\title{
Noise-induced fronts
}

\author{
Miguel A. Santos ${ }^{1}$ and J. M. Sancho ${ }^{2}$ \\ ${ }^{1}$ Departament d'Enginyeria Mecànica, Universitat Rovira i Virgili, Carretera Salou s/n, 43006 Tarragona, Spain \\ ${ }^{2}$ Departament d'Estructura i Constituents de la Matèria, Universitat de Barcelona, Avenida Diagonal 647, 08028 Barcelona, Spain
}

(Received 1 September 1998)

\begin{abstract}
A simple model is introduced that exhibits a noise-induced front propagation and where the noise enters multiplicatively. The invasion of the unstable state is studied, both theoretically and numerically. A good agreement is obtained for the mean value of the order parameter and the mean front velocity using the analytical predictions of the linear marginal stability analysis. [S1063-651X(98)14912-7]
\end{abstract}

PACS number(s): 05.40.-a

\section{INTRODUCTION}

As is well known, nontrivial noise sources in stochastic equations may give rise to a strikingly rich phenomenology representing a drastic contrast with respect to the deterministic (noiseless) behavior [1,2]. The study of the influence of noise in several systems continues being an active field of research, but only very recently has attention been paid to the kind of model that possesses a deterministic global stable ground state that becomes unstable due to the presence of a nontrivial noise source, thus exhibiting a genuine noise induced transition [3-8]. These include those models exhibiting noise induced ordering transitions [6-8], or noise induced patterns [3]. Front dynamics have already been studied under fluctuations but always starting from a deterministic model that itself possesses frontlike solutions [9-13].

In this work we will address the issue of the generation of fronts in such a noisy framework as well as their description, both analytically and numerically. The model is formulated in terms of a stochastic partial differential equation (SPDE) of Langevin type, which contains a multiplicative noisesource term. This kind of noise is in general associated with external fluctuations [1], although internal noise sources may as well give rise to such a coupling with the field under quite general conditions [14]. A standard way for the introduction of an external noise is to let a control parameter of the deterministic model to fluctuate [1].

We will start with a model where in the absence of noise the homogeneous state $\phi(x, t)=0$ is globally stable and thus neither fronts nor any other kind of spatial structures are allowed. Any initial condition will relax to this steady state. We can conjecture whether it would be possible to generate fronts by a kind of coupling with an external noise. We will see that this is the case. So we will have genuine noise induced fronts. This is not a surprise. We have commented before that external multiplicative noise can induce patterns [3] or phases [4,6-8]. We will show here that fronts can also be generated in the same way, explicitly, by the presence of external fluctuations.

In the next section we present the model and the theoretical framework and results. Section III is devoted to the numerical technique and results. Finally, we end with a conclusion and some perspectives of this work.

\section{MODEL AND ANALYTICAL RESULTS}

We start with a generic model of reaction diffusion,

$$
\frac{\partial \phi}{\partial t}=\frac{\partial^{2} \phi}{\partial x^{2}}-\phi\left(a+\phi^{2}\right),
$$

where $a$ is the control parameter [17]. As far as $a \geqslant 0$, the only steady solution of this equation is the homogeneous one with $\phi(x)=0$. Let us allow this parameter to fluctuate as

$$
a \rightarrow a(x, t)=\bar{a}-\epsilon^{1 / 2} \eta(x, t),
$$

where $\eta(x, t)$ is a Gaussian noise of zero mean and correlation given by

$$
\left\langle\eta(x, t) \eta\left(x^{\prime}, t^{\prime}\right)\right\rangle=2 C\left(x-x^{\prime}\right) \delta\left(t-t^{\prime}\right) .
$$

As the noise is white in time the process $\phi(x, t)$ is Markovian. This is not a strong assumption if we consider that the time scale of the noise is much shorter than any other of the field. This spatial part of the correlation function $C\left(x-x^{\prime}\right)$ will be approximated also by a $\delta$ function because we will assume that its correlation length is much more smaller than any other spatial scale of the system. Once we introduce a mesh grid for the spatial domain, this will correspond to taking the correlation length of the noise of the order of the mesh size, $\Delta x$. Thus Eq. (1) becomes a SPDE of the form

$$
\frac{\partial \phi}{\partial t}=\frac{\partial^{2} \phi}{\partial x^{2}}-\phi\left(a+\phi^{2}\right)+\epsilon^{1 / 2} \phi \eta(x, t) .
$$

We will strictly follow the theoretical approach formulated in Ref. [13], based in a former technique [15,16], for describing the effects of the noise by the way of explicitly separating the systematic contribution of the noise in Eq. (4). The main steps are summarized in what follows.

An important point here is the fact that the noise term in Eq. (4) has a nonzero mean value. Using Novikov's theorem [18], and the Stratonovich interpretation, we get

$$
\langle\phi \eta(x, t)\rangle=\epsilon^{1 / 2} C(0)\langle\phi\rangle,
$$

where, $C(0)$ is explicitly given by 


$$
C(0)=\frac{1}{\Delta x}
$$

in the white noise approximation in a lattice.

According to this result we can rewrite Eq. (4) as

$$
\frac{\partial \phi}{\partial t}=\frac{\partial^{2} \phi}{\partial x^{2}}+h(\phi)+\epsilon^{1 / 2} R(\phi, x, t),
$$

where

$$
h(\phi)=-\phi\left(a+\phi^{2}\right)+\epsilon C(0) \phi
$$

and

$$
R(\phi, x, t)=\phi \eta(x, t)-\epsilon^{1 / 2} C(0) \phi .
$$

Taking into account these definitions, the noisy term $R$ has zero mean value and a correlation

$$
\begin{aligned}
\left\langle R(\phi, x, t) R\left(\phi, x^{\prime}, t^{\prime}\right)\right\rangle= & \left\langle\phi(x, t) \eta(x, t) \phi\left(x^{\prime}, t^{\prime}\right) \eta\left(x^{\prime}, t^{\prime}\right)\right\rangle \\
& +O\left(\epsilon^{1 / 2}\right) .
\end{aligned}
$$

Assuming now that the field is expanded as

$$
\phi(x, t)=\phi_{0}(x, t)+\sum_{1}^{\infty} \epsilon^{n / 2} \phi_{n}(x, t),
$$

and, substituting this expression into Eq. (7) we get to the lowest order

$$
\frac{\partial \phi_{0}}{\partial t}=\frac{\partial^{2} \phi_{0}}{\partial x^{2}}-\phi_{0}\left(a^{\prime}+\phi_{0}^{2}\right) .
$$

Details of this theoretical approach are given in Ref. [13], including next order corrections. So we have now that the linear control parameter is renormalized,

$$
a^{\prime}=a-\epsilon C(0) \text {. }
$$

Hence, for those cases in which $a /[\epsilon C(0)]<1$, or, $a^{\prime}<0$, then the homogeneous solution of Eq. (12) $\phi_{0}=0$ is not longer stable and any spatial perturbation will grow until nonlinear terms saturate it. Thus this type of instability will produce a front propagating in both directions if a perturbation of any size is present.

This new state is a spatiotemporal fluctuating field $\phi(x, t)$, and not a smooth front such as it is either in the deterministic case or in the case of Ref. [9]. Nevertheless one can define a kind of mean stationary value $\bar{\phi}_{s t}$ as the nonzero steady state that can be calculated from Eq. (12),

$$
\bar{\phi}_{s t}=\phi_{0(s t)}=[\epsilon C(0)-a]^{1 / 2} .
$$

The linear marginal stability analysis [19] applied to Eq. (12) gives that the front velocity is

$$
\bar{v}=2[\epsilon C(0)-a]^{1 / 2}
$$

and from Eqs. (14) and (15) we have also that

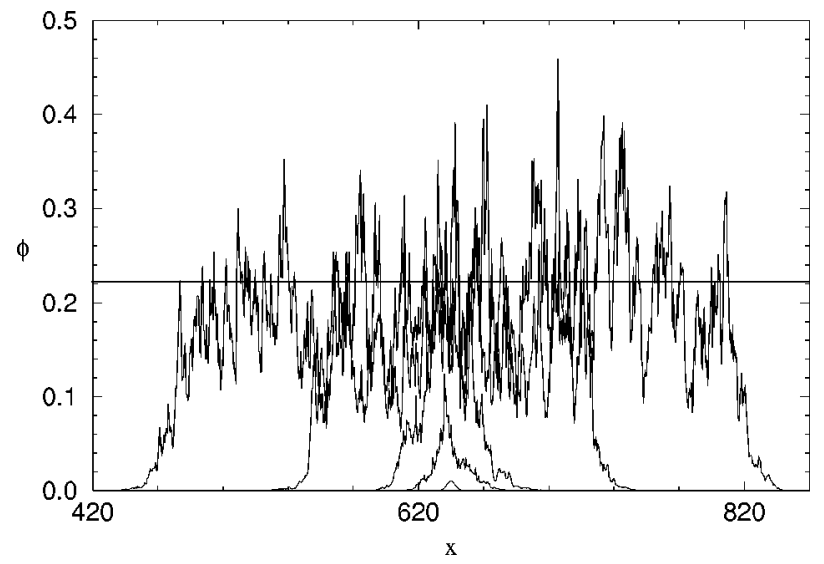

FIG. 1. Initial stages and propagation of a noisy front. The initial Gaussian pulse increases to some saturated value and then gives rise to a front invading the $\phi=0$ state. Snapshots were taken at $t$ $=50,100,240$, and 450. The continuous line shows the expected theoretical value for $\bar{\phi}_{s t}$. Simulations were performed for $a=0.1$ and $\epsilon(0)=0.15$.

$$
\frac{\bar{v}}{\bar{\phi}_{s t}}=2
$$

a curious result that indicates that this quantity is independent of any parameter of the model. These are very simple and precise predictions that we want to check by numerical simulations of Eq. (4).

\section{NUMERICAL PROCEDURE AND RESULTS}

We have numerically integrated our model using a forward propagation scheme in the way of a basic finitedifference Euler algorithm. Periodic boundary conditions have been imposed in a linear system of length $L$, divided in $N$ cells of mesh size $\Delta x$. Simulations were performed for $\Delta x=0.5$ and $\Delta t=0.01$, except were otherwise indicated. For the implementation of the noise source a standard random number generator has been used [20], while the needed Gaussian numbers have been obtained using the algorithm implemented in Ref. [21].

An initial Gaussian-like pulse localized in the center of the system, of height $h=0.01$ and width (mean standard deviation) $w=8 / 3$ has been chosen as a perturbation to favor the development of a front. This perturbation is necessary because the homogeneous initial state $\phi=0$, although unstable, will remain there for ever. Multiplicative noise alone cannot trigger the evolution of a front or any other structure, precisely because it is coupled multiplicatively with the field that is now zero.

When considering the finite difference version of Eq. (4), the noise acquires an effective intensity that is given by

$$
\epsilon(0) \equiv \epsilon / \Delta x=\epsilon C(0) \text {. }
$$

Several front trajectories appear in Fig. 1. Frontlike structure and propagation characteristics are clear. We see how the initial spatial perturbation grows up to some saturated value and after that, the structure formed invades the unstable state $\phi=0$. Moreover, the noise influence is much more apparent here than in those models where their deter- 
ministic part already allows for fronts to rise up [9]. Hence one cannot expect, a priori, better agreements with the theoretical predictions than in those cases. Indeed, as the front is completely induced by the external fluctuations, the steady state is spatiotemporal stochastic, and hence the characterization of the front will deserve a more detailed numerical analysis than in those cases with a deterministic dominant component superimposed on spatiotemporal small fluctuations.

For those systems a common procedure for defining the location of a front propagating into the $\phi=0$ state is via the integral

$$
z(t)=\frac{1}{\bar{\phi}_{s t}} \int_{x_{o}}^{\infty} d x \phi(x, t)=x_{o}+\bar{v} t-\Delta(t)
$$

where $x_{o}$ is an arbitrary constant that can be considered as the location of the initial pulse, $\bar{\phi}_{s t}$ is the steady state left behind the front, and $\bar{v}$ will correspond to the mean propagation velocity of the deterministic effective front [13], which indeed corresponds to the front-end speed of the actual front, while $\Delta(t)$ gives account for the stochastic wandering of the front. As long as we are interested in the evolution of an initial perturbation located somewhere inside the spatial domain, a slightly different numerical approach must be prescribed to evaluate the mean velocity and the mean steady field. In this work, we will concentrate in the case of an initial symmetric perturbation.

The position of the front is defined as

$$
z(t)=\frac{1}{2} L_{\delta}(t)=\int_{L} d x \theta[\phi(x, t)-\delta]
$$

where $\theta(y)$ is the Heaviside step function, and $L_{\delta}(t)$ is the length of the front evaluated as the distance from its two extremes points propagating in opposite directions. Hence $z(t)$ gives the position of the leading part of the half right hand side of the front. We will also consider the area comprised by the front

$$
A(t)=\int_{L} d x \phi(x, t),
$$

where both integrals extend over the whole spatial domain under consideration. $L_{\delta}(t)$ and $A(t)$ are necessary quantities to evaluate the mean velocity $\bar{v}$ and the mean steady state $\bar{\phi}_{s t}$ of the front. It should be borne in mind that some kind of quotient of both magnitudes at late enough times should allow us the evaluation of the $\bar{\phi}_{s t}$. At the same time, the evolution of $L_{\delta}(t)$ will be used to obtain $\bar{v}$.

For the numerical evaluation of $\bar{\phi}_{s t}$ we have considered two possibilities, namely,

$$
\phi(t)=\frac{A(t)}{L_{\delta}(t)},
$$

as well as

$$
\phi(t)=\frac{A(t)-A\left(t_{o}\right)}{L_{\delta}(t)-L_{\delta}\left(t_{o}\right)}, \quad t>t_{o} .
$$

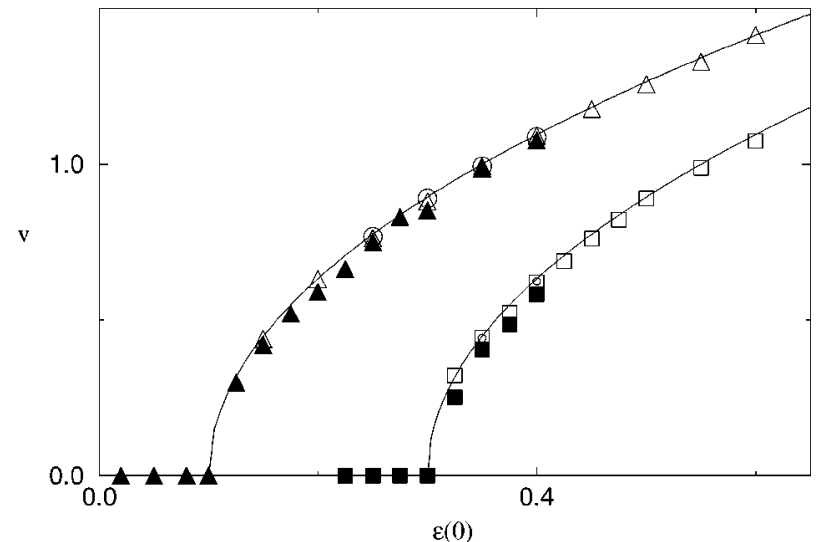

FIG. 2. Front mean velocity vs noise intensity for two values of the order parameter, $a=0.1$ (triangles) and $a=0.3$ (squares). Closed symbols correspond to numerical simulations of the stochastic model (4) for $\Delta x=0.5$ and $\Delta t=0.01$; open triangles and squares stand for $\Delta x=0.1$ and $\Delta t=0.001$. Circles show results for the simulation of the deterministic model (12). Lines show the expected theoretical prediction (15).

In the first case we have to wait for the front to occupy all the domain $L$, provided we have periodic boundary conditions, whereas the second one will allow the determination of $\bar{\phi}_{s t}$ for earlier, although late enough times. However, the first gives better results and was the one used in our simulations.

When the front reaches the boundaries at the time $t_{L}\left[L_{\delta}\left(t_{L}\right) \equiv L\right]$, the sampling of $\phi(t)$ starts. Its stationary value will be calculated as

$$
\bar{\phi}_{s t}=\frac{1}{\left(T-t_{L}\right)} \int_{t_{L}}^{T} d t \phi(t)=\frac{1}{n} \sum_{i=1}^{n} \phi\left(t_{i}\right)
$$

where $n \equiv\left(T-t_{L}\right) / \Delta t$, and $T$ is the time during which the front is let to evolve, always greater than $t_{L}$. This corresponds to an ergodic average of $\phi(t)$.

The mean velocity has been estimated averaging

$$
v(t)=\frac{L_{\delta}(t)-L_{\delta}\left(t_{o}\right)}{2\left(t-t_{o}\right)}, \quad t>t_{o}
$$

between $t=t_{o}+20$ and $t=t_{L}$.

These two definitions give reliable values for $\phi(t)$ $\sim \bar{\phi}_{s t}$, and $v(t) \sim \bar{v}$ if transitory contributions have died out by a proper election of $t_{o}$. The value of $\delta$ in the definition of $L_{\delta}(t)$ has been chosen small enough $(\delta=0.001)$ for it not to become a sensible source of errors.

In Fig. 2 we see the mean front velocity versus the effective intensity of the multiplicative noise. It is clearly seen that for intensities lower than a critical value,

$$
\epsilon_{C}(0)=a,
$$

the velocity is zero, which means that there is no front at all. The agreement with the theoretical prediction (15) is remarkable. Also, $\bar{v}$ turns out to be less sensitive to the discretization scheme of $\Delta x$ and $\Delta t$, than $\bar{\phi}_{s t}$, as we will see later. Some values have been obtained for the deterministic effective front (12), which are shown as four circles for the case $a=0.1$ and two for $a=0.3$ one. For the rest of the symbols 


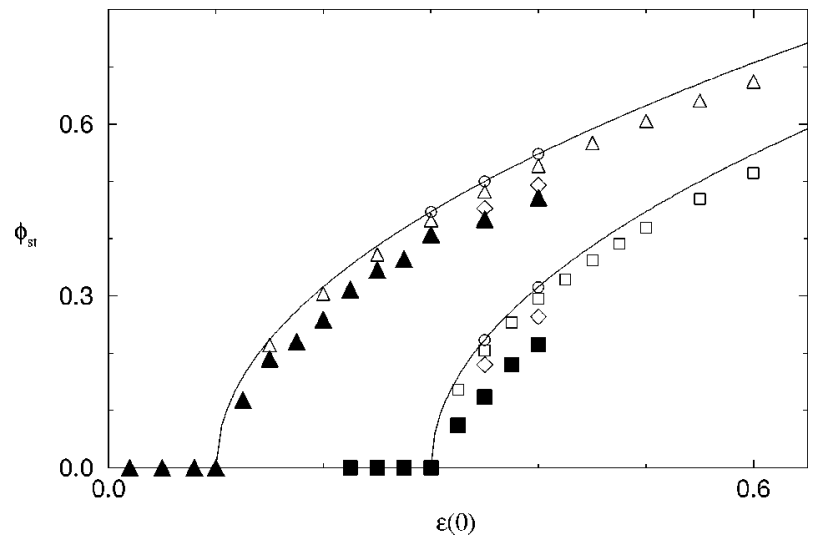

FIG. 3. Mean steady state $\bar{\phi}_{s t}$ vs noise intensity for different values of the control parameter. Diamonds show results for $\Delta x=0.25$ and $\Delta t=0.01$ for each value of $a$. The same notation as in Fig. 2 has been chosen for the rest of the symbols.

their sizes give an upper bound to the error of each point. From now on, each of the symbols will refer to the same values of the parameters as stated for this figure.

Figure 3 presents the mean field $\bar{\phi}_{s t}$ versus the effective noise intensity. We see also that for noise intensities lower than the critical value no front does exist. The theoretical values are sensible greater than the numerical ones, but progressive improvement is achieved by smaller grids $\Delta x$. This effect is clearly seen in this figure. Reducing the mesh size $\Delta x$ for a fixed value of the effective noise intensity $\epsilon(0)$ corresponds to making $\epsilon$ smaller, and the solution of the SPDE [Eq. (4)] will tend to that of the effective deterministic front (12).

In Fig. 4, the ratio of the mean velocity and steady field are plotted versus the effective control parameter $a^{\prime}$. We see that the theoretical prediction (16) is well followed by numerical data. Indeed, the $a=0.3$ values for $\phi(t)$ systematically come up lower than the ones for the case $a=0.1$. This is not so for $v(t)$ where both sets give the same mean value. Hence this shows up in Fig. 4 as a systematically greater value for the case $a=0.3$, and consequently a greater deviation from the expected theoretical value (16). In calculating the error bars, just the fluctuations of the sampled values of $\phi(t)$ and $v(t)$ have been considered. Thus, this does not include possible systematic error that arises from the numerical integration of Eq. (4).

\section{CONCLUSIONS AND PERSPECTIVES}

We conjectured whether it would be possible to generate fronts by a kind of coupling with an external noise. We have

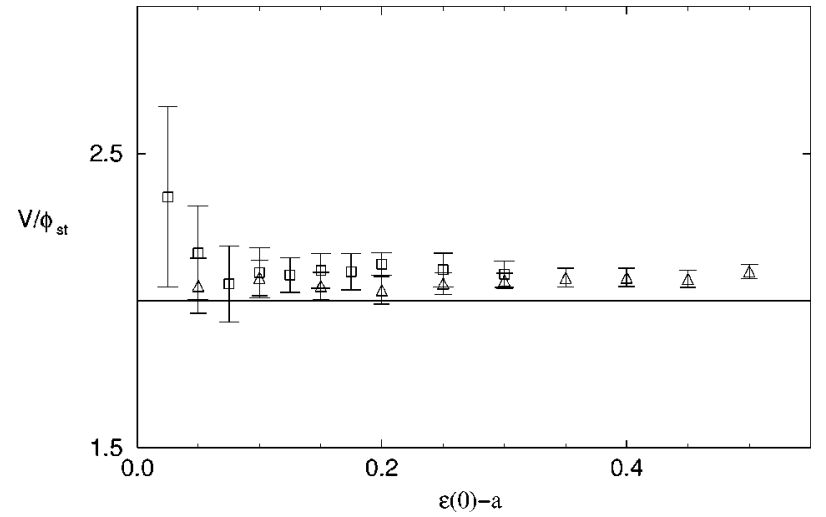

FIG. 4. Ratio of the front mean velocity and steady field vs effective control parameter. See previous figures for symbol notation. The error bars were calculated as the mean standard error for each ergodic average, once the front occupied the whole spatial length $L$.

seen that this is the case. Thus we have shown that fronts can be generated in the same way as other kind of instabilities like patterns or phases, for example. We have studied and characterized the front dynamics of a system that exhibits only one unique stable ground state in its deterministic version, but which undergoes a nonequilibrium transition via a symmetry breaking (stochastic) perturbation in the control parameter.

For the velocity of the front we have obtained perfect agreement with the well known results of linear marginal stability in the presence of noise. The values of $\bar{\phi}_{s t}$ show up to be more sensitive to the mesh steps of the numerical algorithm, but great improvement is achieved by reducing the mesh grid $\Delta x$, in consistency with the results for the velocity.

Due to the symmetry of the model and the fact that the coupling with the noise is linear, only fronts propagating into linearly unstable states are expected. We have also studied a higher-order coupling. If a quadratic contribution is considered in the reaction term then fronts invading a metastable state are possible. Preliminary results confirming the expected possibility of that kind of noise generated front will be presented elsewhere.

\section{ACKNOWLEDGMENT}

This work has been supported by Dirección General de Investigación Científica y Técnica under Project No. PB960241 .
[1] W. Horsthemke and R. Lefever, Noise-Induced Transitions (Springer-Verlag, Berlin, 1984).

[2] R. Benzi, A. Sutera, and A. Vulpiani, J. Phys. A 14, L453 (1981). K. Weisenfeld and F. Moss, Nature (London) 373, 33 (1995).

[3] J. García-Ojalvo, A. Hernández-Machado, and J. M. Sancho, Phys. Rev. Lett. 71, 1542 (1993); J. M. Parrondo, C. Van den
Broeck, J. Buceta, and F. J. de la Rubia, Physica A 224, 153 (1996).

[4] A. Becker and L. Kramer, Phys. Rev. Lett. 73, 955 (1994).

[5] C. Van den Broeck, J. M. R. Parrondo, J. Armero, and A. Hernández-Machado, Phys. Rev. E 49, 2639 (1994).

[6] C. Van den Broeck, J. M. R. Parrondo, and R. Toral, Phys. Rev. Lett. 73, 3395 (1994); C. Van den Broeck, J. M. R. Par- 
rondo, R. Toral, and R. Kawai, Phys. Rev. E 55, 4084 (1997).

[7] J. García-Ojalvo, J. M. R. Parrondo, J. M. Sancho, and C. Van den Broeck, Phys. Rev. E 54, 6918 (1996); S. Kim, S. H. Park, and C. S. Ryu, Phys. Rev. Lett. 78, 1616 (1997); R. Müller, K. Lippert, A. Kühnel, and U. Behn, Phys. Rev. E 56, 2658 (1997).

[8] W. Genovese, M. A. Mun̂z, and J. M. Sancho, Phys. Rev. E 57, R2495 (1998).

[9] J. Armero, J. M. Sancho, J. Casademunt, A. M. Lacasta, L. Ramírez-Piscina, and F. Sagués, Phys. Rev. Lett. 76, 3045 (1996).

[10] G. F. Mazenko, O. T. Valls, and P. Ruggiero, Phys. Rev. B 40, 384 (1989).

[11] A. Lemarchand, A. Lesne, and M. Mareschal, Phys. Rev. E 51, 4457 (1995).

[12] J. Riordan, C. R. Doering, and D. ben-Arraham, Phys. Rev. Lett. 75, 565 (1995).

[13] J. Armero, J. Casademunt, L. Ramírez-Piscina, and J. M. Sancho, Phys. Rev. E 58, 5494 (1998).

[14] L. Ramírez-Piscina, A. Hernández-Machado, and J. M. San- cho, Phys. Rev. B 48, 119 (1993).

[15] H. Malchow and L. Schimansky-Geir, Noise and Diffusion in Bistable Nonequilibrium Systems (Teubner - Texte zur Physik, Berlin, 1985).

[16] F. de Pasquale, J. Gorecki, and J. Popielawski, J. Phys. A 25, 433 (1992).

[17] R. A. Fisher, Ann. Eugen. 7, 355 (1937); A. Kolmogorov, I. Petrovsky, and N. Piskunov, Bulletin de l'université d'état à Moscou, Sère internationale, Sec. A, 1, 1 (1937) translated in P. Pelcé, Dynamics of Curved Fronts. Perspectives in Physics, edited by H. Araki, J. Libchaber, and G. Parisi (Academic Press, San Diego, 1988).

[18] E. A. Novikov, Zh. Eksp. Teor. Fiz. 47, 1919 (1964) [Sov. Phys. JETP 20, 1290 (1965)].

[19] W. Saarlos, Phys. Rev. A 39, 6367 (1989).

[20] Marsaglia and Zaman, Report No. FSU-SCRI-87-50 (unpublished). Actually we use a slightly modified version due to $\mathrm{F}$. James, Comput. Phys. Commun. 60, 329 (1990).

[21] R. Toral and A. Chakrabarti, Comput. Phys. Commun. 74, 327 (1991). 